\title{
Changes of Membrane Lipids in Apple Buds During Dormancy and Budbreak
}

\author{
Shiow Y. Wang and Miklos Faust \\ Fruit Laboratory, Beltsville Agricultural Research Center, Agricultural Research Service, U.S. \\ Department of Agriculture, Beltsville, MD 20705
}

Additional index words. Malus domestics, fatty acids, glycolipid, phospholipid, sterols

\begin{abstract}
The changes of membrane lipids in apple (Malus domestics Borkh. cv. Delicious) auxillary and terminal buds from August to April were determined. The predominant lipids were monogalactosyl diglyceride (MGDG), digalactosyl diglyceride (DGDG), phosphatidylcholine (PC), and phosphatidylethanolamine (PE). An increase in membrane polar lipids was associated with budbreak and bud growth from August to April. Linolenic acid was the predominant fatty acid in MGDG, DGDG, and PC, while linoleic acid was predominant in PE. Phosphatidylglycerol (PG) and phosphatidylinositol (PI) contained a high amount of palmitic acid. The ratio of (18:2 + 18:3) to 18:1 fatty acids in galactolipids in apple buds increased from August to April. B-Sitosterol and sitosteryl ester were the predominant sterols in apple buds. An increase in sitosterol, a decrease in sitosteryl ester, and a decline in the ratio of free sterols to phospholipids occurred during budbreak in spring. A decrease in sitosterol was associated with bud expansion in spring.
\end{abstract}

Temperate fruit trees require a period of low temperature in winter to break bud dormancy. Temperature during growth influences membrane fatty acid composition and unsaturation (Lyons, 1973). The physical state of the membrane lipids plays an important role in determining the physiological function of plant tissue (Brenner, 1984; Raison and Chapman, 1976; Wang, 1982). Lipid molecules are essential building blocks for every membrane of the living cell, and membranes are sites for many specific enzymatic activities, transport ions and metabolizes, and hormonal receptors (Brenner, 1984). Certain plant hormones and growth regulators can substitute for chilling to induce budbreak (Wang and Faust, 1988). The plant bioregulants thidiazuron and nitroguanidines released lateral buds from dormancy and induced an increase in galacto- and phospholipids and the ratio of unsaturated fatty acids to saturated fatty acids (Wang and Faust, 1988, 1989a, 1989b). A decrease in the percentage of the sitosterol and sitosteryl ester was accompanied by an increase in campesterol and stigmasterol at the beginning of rapid growth. A decrease in the ratio of free sterol to phospholipids and an increase in the ratio of campesterol + stigmasterol to sitosterol upon breaking dormancy was also found in apple buds induced by thidiazuron and nitroguanidines (Wang and Faust, 1988, 1989a, 1989b). However, no information is available about the changes of membrane lipids under natural growing conditions during budbreak and bud growth of deciduous fruit trees. The objectives of this research are to quantify the changes of the membrane lipids during natural growing conditions and to determine whether they are similar to those induced by growth regulators as reported by Wang and Faust (1988, 1989a, 1989b). This information would be helpful in understanding the role of membrane lipids in breaking dormancy and in improving the regulation of the time of budbreak.

\section{Materials and Methods}

Plant material. Twenty 10-year-old 'Delicious' apple trees planted at the Agricultural Research Center in Beltsville, Md.,

Received for publication 23 Jan. 1990. We wish to thank Amy L. Jordan for technical assistance. Use of a company or product name does not constitute an endorsement by USDA nor imply approval to the exclusion of other suitable products. The cost of publishing this paper was defrayed in part by the payment of page charges. Under postal regulations, this paper therefore must be hereby marked advertisement solely to indicate this fact. were used in this study. Twenty newly produced shoots (1-yearold branches) were randomly collected from each tree between 8:30 and 9:30 AM on the 15th of each month from Aug. 1987 to Mar. 1988. Shoots were also collected on 5 and 22 Apr. Terminal buds and the first seven axillary buds of each shoot were separated and used in this study. Triplicate axillary and terminal bud samples of $1.0 \mathrm{~g}$ fresh weight were collected from these shoots at each sampling time.

Extraction, fractionation, and analysis of polar lipids. The extraction, fractionation, and analysis of lipids in apple buds were described by Wang and Faust (1989a, 1989b). Purified lipids were separated into neutral, glyco-, and phospholipid fractions by silicic-acid column chromatography on 100- to 200mesh Bio Sil A (Bio Rad Laboratories, Richmond, Calif.). The glycolipid and phospholipid fractions were further separated by thin layer chromatography (TLC) on $20 \times 20$-cm glass plates precoated with a $250 \mu \mathrm{m}$ thickness of silica gel 60 (EM Reagents, Darmstadt, FRG) using 100 acetone : 2 acetic acid : 1 water (by volume) and 85 chloroform : 15 methanol : 10 acetic acid : 3.5 water (by volume), respectively. Individual galactolipids and phospholipids were identified by cochromatography with authentic standards (Sigma, St. Louis, and Supelco, Bellefonte, $\mathrm{Pa}$.) and by detection with spray reagents specific for hexose sugars or phosphate. Individual lipid bands were scraped off and eluted in 2 chloroform : 1 methanol $(\mathrm{v} / \mathrm{v})$. Total fatty acids esterified to polar lipids from apple buds were derivatized to fatty acid methyl esters (FAME) for flame ionization detection-gas chromatography (FID-GC) analysis (Wang and Faust, 1988). n-Heptadecanoic acid was included in all samples as an internal standard, and methyl heptadecanoate was used as an external standard. Individual FAME were identified by a comparison of retention times with those of authentic standards (Supelco). This tentative identification of major polar lipid fatty acids was corroborated by further analysis of the FAME by gas chromatography-mass spectrometry (GC-MS) (Wang et al., 1988).

The neutral lipid fraction from apple buds was dried under a stream of $\mathrm{N}_{2}$ and redissolved in $2 \mathrm{ml}$ of hexane. Silicic-acid column chromatography (100- to 200-mesh Bio Sil A, Bio Rad

Abbreviations: DGDG, digalactosyl diglyceride; MGMG, monogalactosyl diglyceride; PC, phosphatidylcholine; PE, phosphatidylethanolamine; PG, phosphatidylglycerol; PI, phosphatidylinositol. 
Laboratories) was used to separate the steryl ester and free sterols by elution with 10 hexane : 1 ether (v/v) and 2 hexane : 1 ether (v/v), respectively. Following the addition of $20 \mu \mathrm{g}$ of cholesterol or cholesterol myristate as an internal standard, steryl ester was saponified in $1.0 \mathrm{ml}$ of $1 \mathrm{~N} \mathrm{KOH}$ in $85 \%$ ethanol for $1 \mathrm{hr}$ at $80 \mathrm{C}$ (under $\mathrm{N}_{2}$ ). Free sterols were precipitated with digitonin (Sigma). The digitonides were collected on a glass fiber filter (Reeve Angel, Clifton, N. J.) and washed with 1 acetone : 2 diethyl ether $(\mathrm{v} / \mathrm{v})$. Free sterols were liberated from the digitonides by refluxing in $1 \mathrm{ml}$ of pyridine for $30 \mathrm{~min}$ at 100C. After cooling, $1 \mathrm{ml}$ of deionized, distilled water was added, and the free sterols were recovered by extraction with 2 $\mathrm{ml}$ of hexane. Sterol composition was determined by FID-GC and further confirmed by GC-MS (Wang et al., 1988). Data were tested with analysis of variance procedures. All values are the means of three replicate samples. All LSD values are at $P=0.05$. Standard errors of means are reported in Tables 1 and 2.

\section{Results and Discussion}

Bud development. The axillary and terminal bud dry weight stayed almost constant from August to February, when growth was extremely slow (Fig. 1). Bud dry weight started to increase in March and a rapid increase was noticed in April, which coincided with the time of bud expansion and renewed growth. The terminal buds were about three times as large as the axillary buds in August, and $\approx 1.4$ times as large when buds were collected in late April (Fig. 1).

The scales of buds became increasingly pigmented from $\mathrm{Au}-$ gust to October. The external appearance of the buds did not change during winter. Leaf primordia and the meristem grew with bud swell in spring, while the outer bud scale abscised.

Galactolipids. Galactolipids were among the major lipids of apple buds (axillary and terminal). MGDG was the major galactolipid, and the MGDG : DGDG ratio was $\approx 4.0$ in apple buds [Tables 1 and 2). MGDG and DGDG are the major chloroplast thylakoid lipids (Williams et al., 1983). Chloroplast development is accompanied by an increased synthesis of galactosyldiglycerides. They play an essential role in the maintenance of electron transport systems (Williams et al., 1983), fatty acid synthesis (Sellden and Selstam, 1976), and photo-

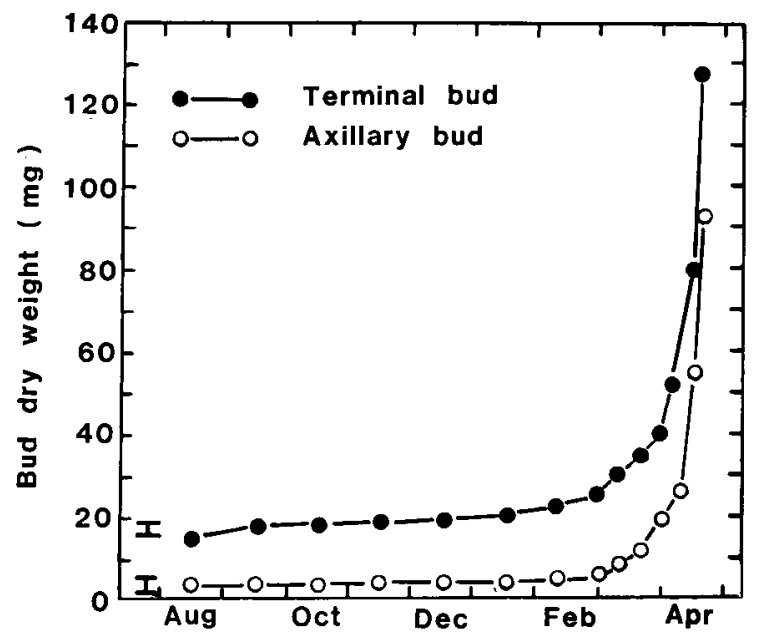

Fig. 1. Changes in dry weight of apple axillary and terminal buds from August to April. reduction of cytochrome (Chang and Lundin, 1965). They may also be involved in carbohydrate transport (Benson, 1964). An increase in the galactolipids of the axillary and terminal buds was observed as the season progressed from August to April (Tables 1 and 2). A sharp increase was found in late April, which coincided with the time of bud expansion (Tables 1 and 2 ). Thus, increases in these lipids were correlated with the accelerated development of chloroplasts in apple buds.

The galactolipids MGDG and DGDG were relatively unsaturated compared to the other lipids. The ratio of $(18: 2+$ 18:3) to 18:1 in apple buds increased from August to April (Fig. 2). The sharp increase of this ratio in April indicates that galactolipids had more fatty acids, with high degrees of unsaturation during budbreak and bud development. The fatty acid profiles of galactolipids from axillary and terminal buds were similar. Palmitic (C16:0), stearic (C18:0), oleic (C18:1), linoleic (C18:2), and linolenic acid (C18:3)were major components of galactolipids in the buds. Linolenic acid was the predominant fatty acid in MGDG and DGDG (Figs. 3 and 4). The proportion of 18:3 in MGDG was much greater than in DGDG. The amount of palmitic, stearic, oleic, and linoleic acid decreased, whereas linolenic acid increased from August to April (Figs. 3 and 4).

Phospholipids. Phospholipids are known to be functional components of the cell membrane and of membranes of various cell organelles (Radunz, 1986). The lipid composition of the membrane obtained from apple buds was also dominated by phospholipids. Tables 1 and 2 show seasonal changes of the individual phospholipids, PC, PE, PG, and PI in apple axillary and terminal buds. Phosphatidic acid was a very minor component in all samples, suggesting minimal phospholipid hydrolysis by phospholipase D (Mazliak, 1973). PC and PE represented a major portion of the phospholipids. Among the individual phospholipids, PC, PE, PI, and PG accounted for $\approx 52 \%, 24 \%, 17 \%$, and $7 \%$, respectively (Tables 1 and 2 ). The amount of individual and total phospholipids in the axillary and terminal buds was similar. An increase in the phospholipids PC, PG, PE, and PI of the buds was associated with bud growth from August to April. The amount of increase was different for each lipid. PC is the major phospholipid of the chloroplast envelope and plays an important role in lipid transport between organelles and tends to maintain a bilayer configuration under various physiological conditions (Block et al., 1983; Gagne et al., 1985). It seems likely that an accumulation of PC in membranes during cold winter months could be part of a common mechanism that results in increased membrane fluidity and prevents the formation of the nonbilayer lipid phase under low winter temperature. Palmitic (C16:0), stearic (C18:0), oleic (C18:1), linoleic (C18:2), and linolenic (C18:3) were major fatty-acyl constituents in each of the four phospholipids from apple buds. The percentages of individual fatty acids in the various phospholipids of the axillary and terminal buds were similar (Figs. 5-8). The seasonal changes in fatty acid composition differed somewhat between the individual phospholipids. The ratio of $(18: 2+18: 3)$ to $18: 1$ fatty acids of phospholipids in apple buds increased from August to budbreak (Fig. 2). Fatty acid unsaturation is another key factor related to membrane function. Its principal effect is to create fluid environments in the membrane so that proteins and lipids can diffuse in the plane of the membrane and so that proteins can undergo confirmational changes during interaction with ligands, substrates, or effecters (Kimelberg, 1977). Fatty acids can influence phase-transition temperatures of membrane lipids and the 
Table 1 . Seasonal change $\left(\mu \mathrm{g} \cdot \mathrm{g}^{-1}\right.$ dry weight) of glycolipids and phospholipids in axillary buds of apple trees.'

\begin{tabular}{|c|c|c|c|c|c|c|}
\hline \multirow[b]{2}{*}{ Month } & \multicolumn{2}{|c|}{ Glycolipid } & \multicolumn{4}{|c|}{ Phospholipid } \\
\hline & MGDG & DGDG & $\mathrm{PC}$ & $\mathrm{PG}$ & $\mathrm{PE}$ & PI \\
\hline August & $54 \pm 4$ & $11 \pm 2$ & $122 \pm 6$ & $12 \pm 2$ & $36 \pm 2$ & $52 \pm 3$ \\
\hline September & $61 \pm 3$ & $14 \pm 1$ & $148 \pm 8$ & $14 \pm 3$ & $48 \pm 5$ & $77 \pm 5$ \\
\hline October & $69 \pm 4$ & $18 \pm 2$ & $168 \pm 10$ & $22 \pm 3$ & $76 \pm 9$ & $80 \pm 4$ \\
\hline November & $69 \pm 3$ & $19 \pm 2$ & $244 \pm 13$ & $40 \pm 4$ & $125 \pm 7$ & $81 \pm 6$ \\
\hline December & $93 \pm 8$ & $23 \pm 1$ & $283 \pm 11$ & $48 \pm 5$ & $149 \pm 10$ & $98 \pm 8$ \\
\hline January & $97 \pm 7$ & $24 \pm 2$ & $292 \pm 14$ & $52 \pm 6$ & $194 \pm 8$ & $100 \pm 7$ \\
\hline February & $111 \pm 6$ & $25 \pm 1$ & $643 \pm 26$ & $77 \pm 7$ & $206 \pm 6$ & $121 \pm 5$ \\
\hline March : & $140 \pm 9$ & $35 \pm 4$ & $993 \pm 38$ & $87 \pm 4$ & $228 \pm 11$ & $123 \pm 4$ \\
\hline April (early) & $214 \pm 11$ & $58 \pm 8$ & $1171 \pm 42$ & $153 \pm 5$ & $371 \pm 18$ & $229 \pm 11$ \\
\hline April (late) & $1357 \pm 49$ & $233 \pm 11$ & $1563 \pm 51$ & $409 \pm 11$ & $552 \pm 19$ & $361 \pm 14$ \\
\hline
\end{tabular}

${ }^{z}$ Mean of three replicates $\pm S E$.

Table 2. Seasonal change ( $\mu \mathrm{g} \cdot \mathrm{g}^{-1}$ dry weight) of glycolipids and phospholipids in terminal buds of apple trees.

\begin{tabular}{|c|c|c|c|c|c|c|}
\hline \multirow[b]{2}{*}{ Month } & \multicolumn{2}{|c|}{ Glycolipid } & \multicolumn{4}{|c|}{ Phospholipid } \\
\hline & MGDG & DGDG & $\mathrm{PC}$ & $\mathrm{PG}$ & PE & $\mathrm{PI}$ \\
\hline August & $51 \pm 3$ & $15 \pm 1$ & $109 \pm 7$ & $14 \pm 1$ & $56 \pm 5$ & $88 \pm$ \\
\hline September & $53 \pm 4$ & $17 \pm 3$ & $125 \pm 9$ & $20 \pm 2$ & $109 \pm 8$ & $89 \pm$ \\
\hline October & $70 \pm 4$ & $18 \pm 2$ & $207 \pm 11$ & $39 \pm 4$ & $183 \pm 11$ & $99 \pm$ \\
\hline Nor & $74 \pm 3$ & $19 \pm 3$ & $243 \pm 14$ & $44 \pm 3$ & $196 \pm 12$ & $103 \pm 2$ \\
\hline $\mathrm{D}$ & $80 \pm 2$ & $19 \pm 2$ & $274 \pm 17$ & $46 \pm 2$ & $203 \pm 6$ & $107 \pm 4$ \\
\hline $\mathrm{Jg}$ & $92 \pm 5$ & $20 \pm 2$ & $580 \pm 20$ & $48 \pm 4$ & $207 \pm 4$ & $109 \pm 3$ \\
\hline 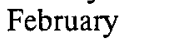 & $102 \ddagger 4$ & $26 \pm 3$ & $604 \pm 8$ & $48 \pm 6$ & $216 \pm 9$ & $118 \pm 4$ \\
\hline March & $108 \pm 8$ & $33 \pm 6$ & $974 \pm 38$ & $69 \pm 4$ & $377 \pm 21$ & $119 \pm$ \\
\hline April (early) & $143 \pm 11$ & $47 \pm 4$ & $1067 \pm 40$ & $78 \pm 3$ & $413 \pm 15$ & $160 \pm 12$ \\
\hline April (late) & $693 \pm 26$ & $542 \pm 17$ & $1447 \pm 42$ & $374 \pm 11$ & $633 \pm 21$ & $374 \pm 19$ \\
\hline
\end{tabular}

${ }^{z}$ Mean of three replicates $\pm \mathrm{SE}$.

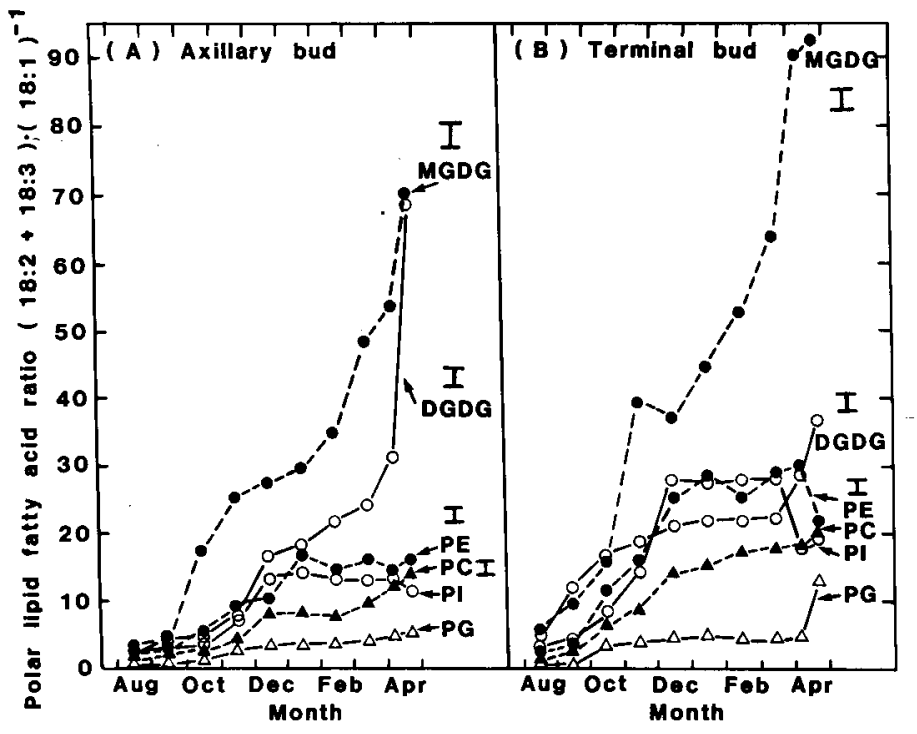

Fig. 2. Fatty acid saturation of apple bud polar membrane lipids during growing season from August to April.

activities of membrane-bound enzymes and transport proteins (Kimelberg, 1977; Sandermann, 1978).

In general, linolenic acid (C18:3) was the major C18 fatty acid in PC, while C18:2 was predominant in PE (Figs. 5 and $6)$. The relative percentage of 18:3 increased along with decreases in relative percentages of $18: 1$ in both $\mathrm{PC}$ and $\mathrm{PE}$ from August to April. Linoleic acid (C18:2) in PC and PE showed sharp increases, while palmitic acid (C16:0) content decreased

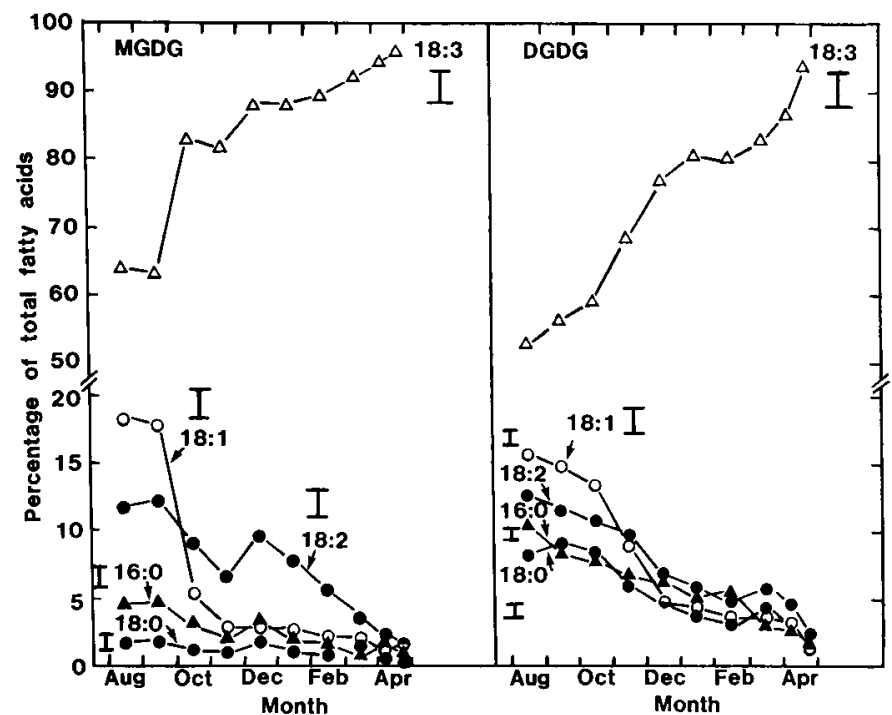

Fig. 3. Fatty acid composition (weight percent of total) of MGDG and DGDG in apple axillary buds during growing season from $\mathrm{Au}-$ gust to April.

from August to budbreak. Linoleic acid (C18:2) decreased and C16:0 increased after budbreak and during bud growth in spring. The relative percentage of 18:0 in $\mathrm{PC}$ and $\mathrm{PE}$ remained low and constant from August to April (Figs. 5 and 6). PG is largely confined to the chloroplasts. PI is present in all plant membranes and generally comprises $1 \%$ to $10 \%$ of the acyl lipids (Hardwood, 1980). PG and PI contained a relatively higher amount 


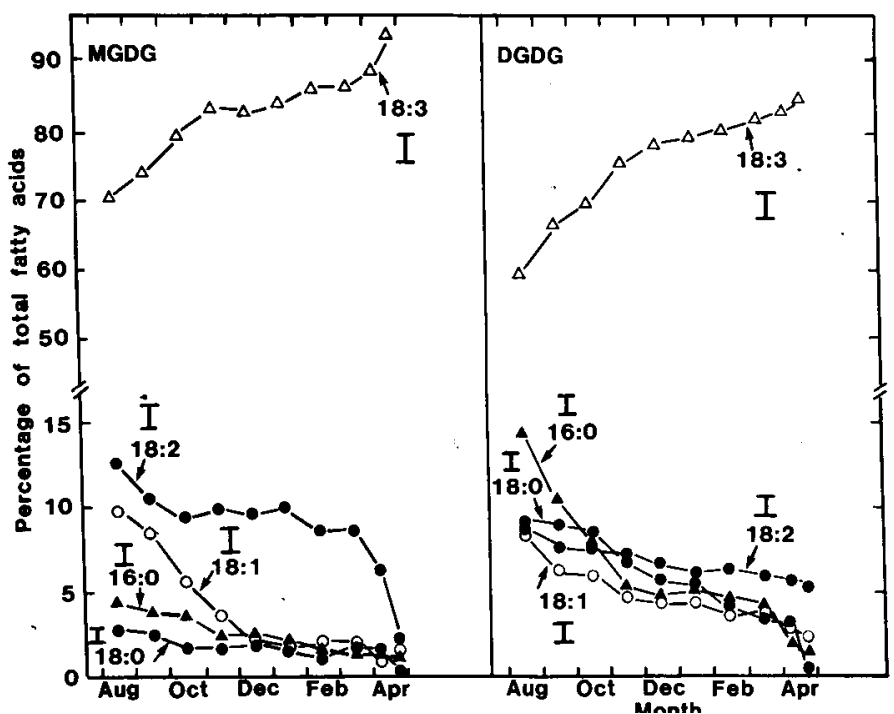

Fig. 4. Fatty acid composition (weight percent of total) of MGDG and DGDG in apple terminal buds during growing season from $\mathrm{Au}-$ gust to April.

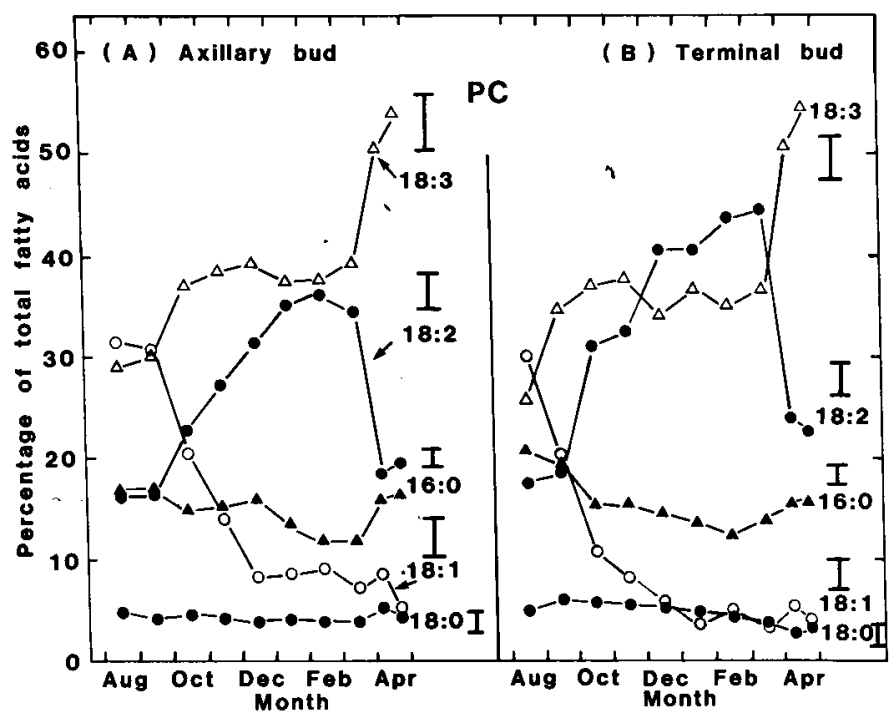

Fig. 5. Fatty acid composition (weight percent of total) of PC in apple buds during growing season from August to April.

of the saturated fatty acid, palmitic acid (16:0) (Figs. 7 and 8). The relative percentage of 16:0 and 18:0 in PI did not change significantly from August to April; however, a decrease of 16:0 was found in PG. The relative content of 18:2 and 18:3 in PG and PI was increased at the expense of 18:1 from fall to winter. In spring, a sharp decrease of 18:2 and an increase of 18:3 were seen in PI and PG, suggesting that PI and PG are most likely involved in the desaturation of $18: 2$ to $18: 3$. Small amounts of oleic acid (18:0) were found in PI and PG and remained constant throughout the growing season (Figs. 7 and 8). All these changes may simultaneously increase overall membrane fluidity and preserve the physiological bilayer phase for budbreak and bud growth in apple trees.

Sterols. Sitosterol is the major desmethylsterol in apple buds (Fig. 9). It is the most widely distributed sterol in higher plants and has 29 carbon atoms. Campesterol and stigmasterol were present only in trace amounts (data not shown). An increase in free sterol content coincided with the time of budbreak and bud

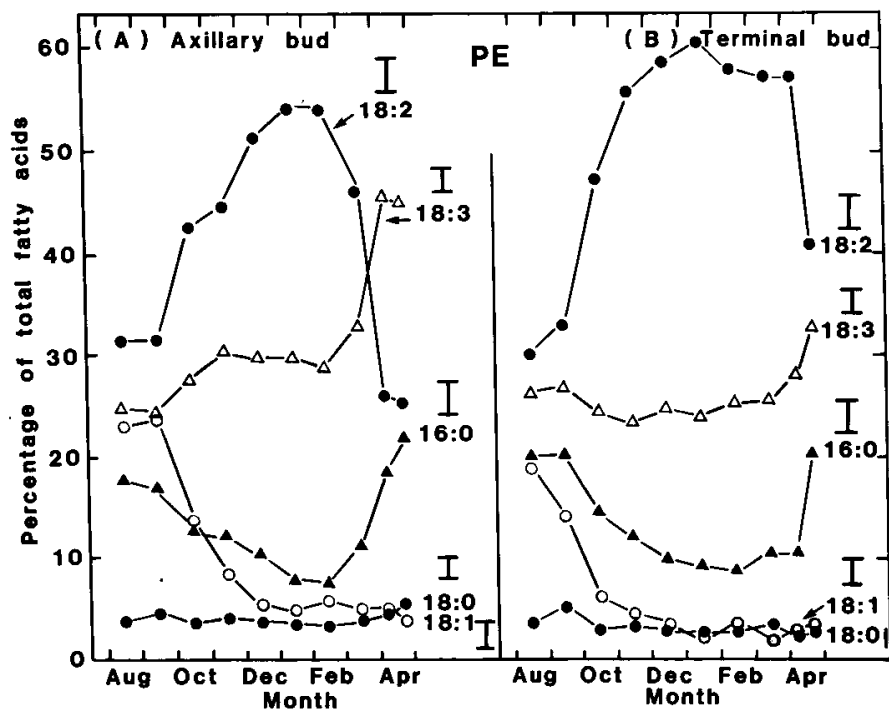

Fig. 6. Fatty acid composition (weight percent of total) of PE in apple buds during growing 'season from August to April.

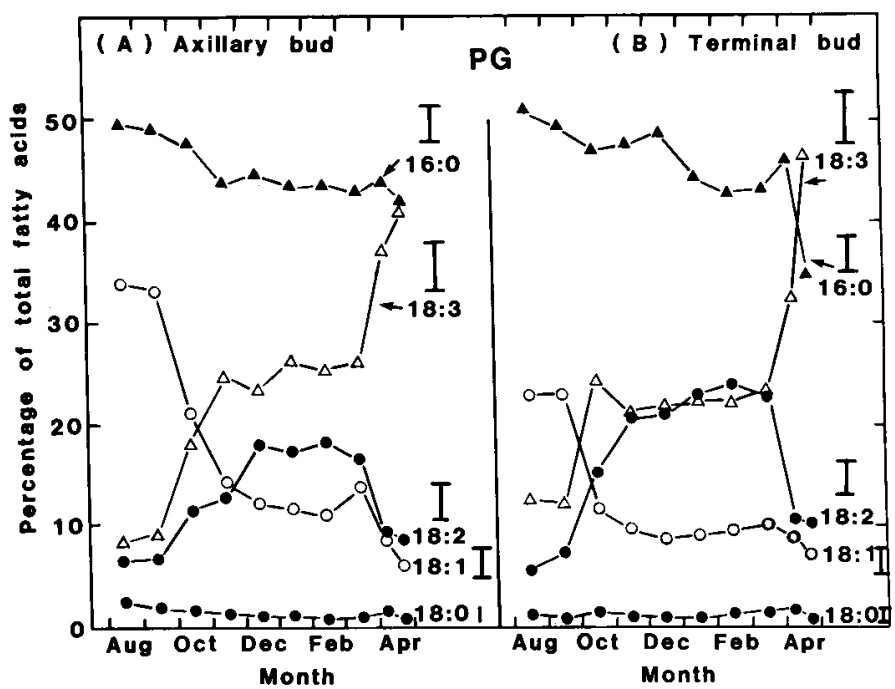

Fig. 7. Fatty acid composition (weight percent of total) of $P G$ in apple buds during growing season from August to April.

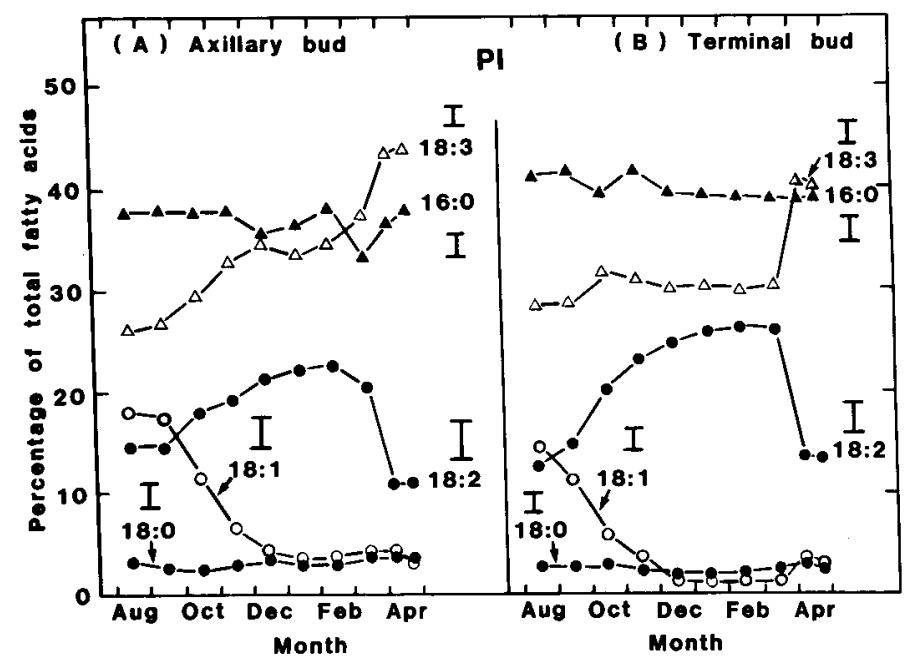

Fig. 8. Fatty acid composition (weight percent of total) of PI in apple buds during growing season from August to April.

J. Amer. Soc. Hort. Sci. 115(5):803-808. 1990. 


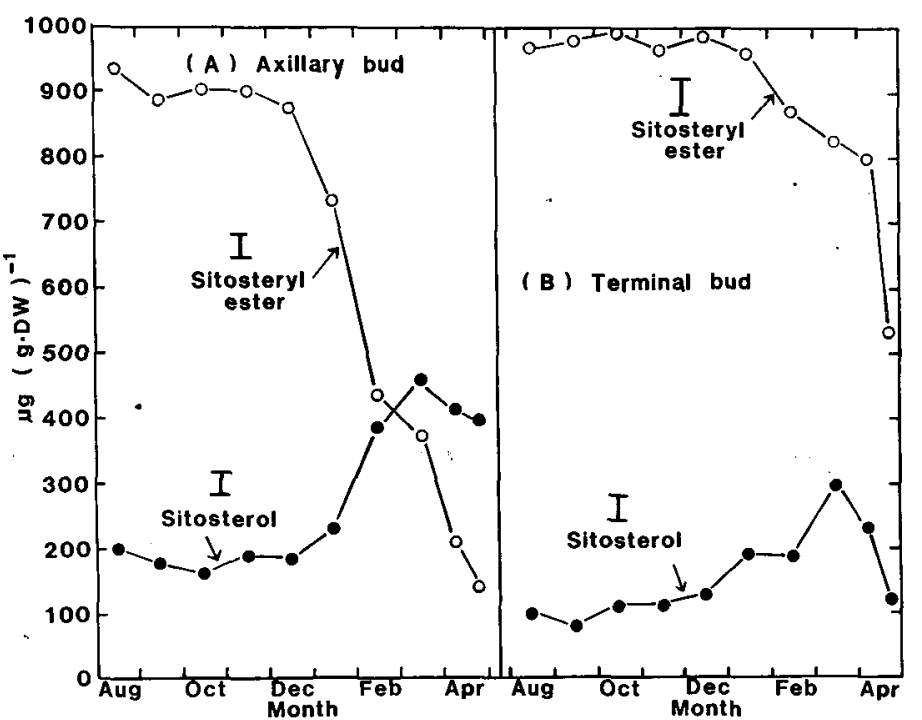

Fig. 9. Changes of sitosterol and sitosteryl ester in apple axillary and terminal buds during growing season from August to April.

expansion in early spring (Fig. 9). This increase indicates that sterol synthesis is rapid in actively growing and differentiating plant tissues (Geuns, 1973). Dormant buds contained a considerable proportion of the sterol in the esterified form. The steryl ester content of buds fell significantly at the end of dormancy and remained at a low level during budbreak and bud growth, while the free sterol contents increased. The increase in free sterols may be, in part, the result of the hydrolysis of reserve steryl esters in buds. Steryl esters in buds may play a role as storage reserve to supply the sterols required to meet the demands of the developing bud for membrane assembly at the onset of budbreak and bud growth. The use of free sterols during rapid bud expansion and renewed growth in April for synthesizing cellular constituents may cause the decrease in sterol content (Fig. 9).

Sterols may affect membrane properties directly by insertion in the membrane or indirectly through their effects in lipid metabolism. Sterols and steryl esters may also be involved in some regulatory processes in phytosterol production (Goad, 1983; Grunwald, 1975). A high content of sterols and sterol conjugates is characteristic for the plasma membrane of the plant cell (Heftmann, 1971; Goad, 1983; Grunwald, 1975). These steryl lipids serve to stabilize the lipid bilayer (Kuiper, 1985). The environmental factors may affect the sterol and steryl ester composition of plants. Changes in sterol content and composition have been correlated with altered membrane permeability and development in higher plants (Goad, 1983; Grunwald, 1975).

The sterols : phospholipids ratio is an index of the fluidity of the membrane (Quinn, 1981). A decrease in this ratio will initiate changes in the fluidity of the membranes. The ratio of free sterols to total phospholipids in apple buds decreased from $\mathrm{Au}$ gust to April (Fig. 10), primarily due to the large increase in phospholipids. The ratio was at its lowest during bud expansion in late April. This relationship may indicate an increase in membrane fluidity and cell metabolism, a probable prerequisite for budbreak and bud growth in spring.

\section{Conclusion}

The seasonal changes of membrane lipids in apple buds from dormancy in winter to budbreak and bud growth in spring appeared to be similar to the general pattern of lipid metabolism

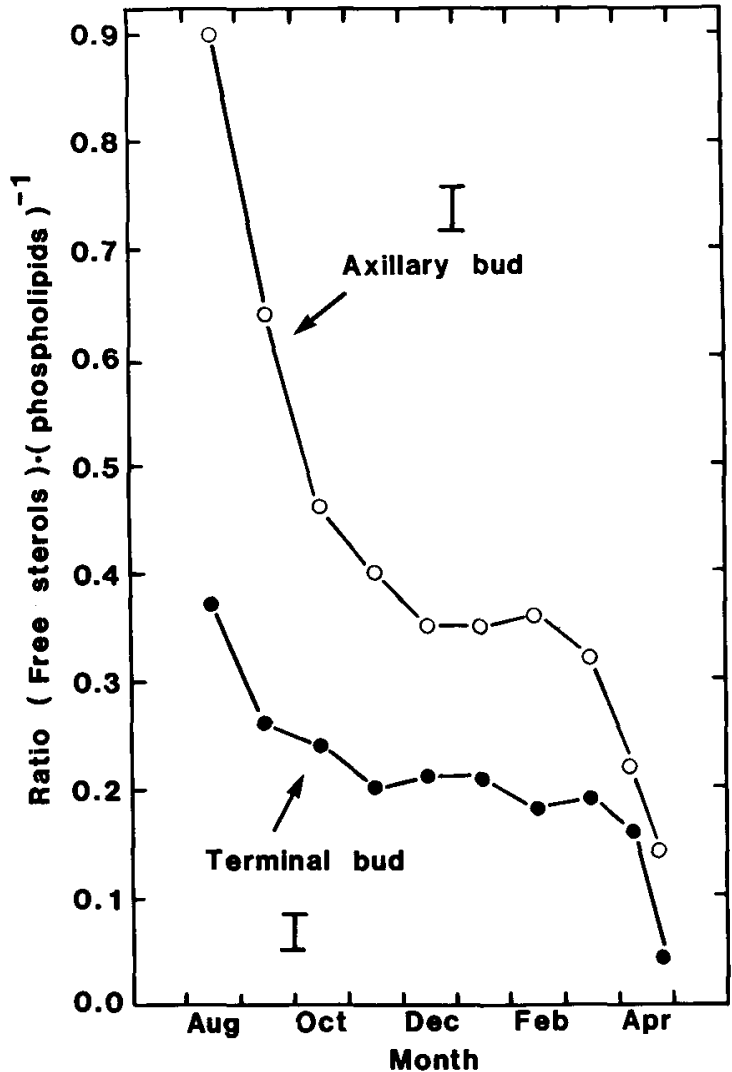

Fig. 10. Changes of the free sterols : phospholipids ratio in apple axillary and terminal buds during growing season from August to April.

induced by the plant growth regulators thidiazuron and nitroguanidine (Wang and Faust, 1988, 1989a, 1989b). Apple buds exposed to low winter temperatures responded by increasing the degree of unsaturation of the fatty acids of their membrane lipids, changing polar head group composition, increasing membrane phospholipid content, and changing sterol levels and composition. The ratio of sterols to phospholipids decreased during budbreak and bud growth in the spring. This study provides evidence that metabolic activities in dormant buds continue at a slow rate and that changes in membrane lipid composition can lead to conditions favorable for budbreak at the end of winter and rapid bud growth in the spring.

\section{Literature Cited}

Block, M. A., A.J. Dome, J. Joyard, and R. Deuce. 1983. Preparation and characterization of membrane fractions enriched in outer and inner envelope membranes from spinach chloroplasts. II. Biochemical characterization. J. Biol. Chem. 258:13281-13286.

Benson, A.A. 1964. Plant membrane lipids. Annu. Rev. Plant Physiol. 15:1-16.

Brenner, R.R. 1984. Effect of unsaturated acids on membrane structure and enzyme kinetics. Progress Lipid Res. 23:69-96.

Chang, S.B. and K. Lundin. 1965. Specificity of galactolipids in photochemical reaction coupled with cytochrome $\mathrm{c}$ reduction. Biochem. Biophys. Res. Commun. 21:424-431.

Gagne, J., L. Stamatatos, T. Diacovo, S.W. Hui, P.L. Yeagle, and J.R. Silvius. 1985. Physical properties and surface interactions of bilayer membranes contained $\mathrm{N}$-methylated phosphatidylethanolamines. Biochemistry 24:4400-4408.

Geuns, J.M.C. 1973. Variations in sterol composition in etiolated mung bean seedlings. Photochemistry 12:103-106. 
Goad, L.J. 1983. How is sterol synthesis regulated in higher plants? Biochem. Sot. Trans. 11:548-552.

Grunwald, C. 1975. Plant sterols. Annu. Rev. Plant Physiol. 26:209236.

Harwood, J-L. 1980. Plant acyl lipids: Structure, distribution, and analysis, p. 1-55. In: P.K. Stumpf (cd.). The biochemistry of plants. vol. 4. Lipids: Structure and function. Academic, New York. .

Heftmann, E. 1971. Functions of sterols in plants. Lipids 6:128-133.

Kimelberg, H.K. 1977. The influence of membrane fluidity on the activity of membrane-bound enzymes, p. 205-293. In: G. Poste and G.L. Nicolson (eds.). Dynamic aspects of cell surface organization. North-Holland, New York.

Kuiper, P.J.C. 1985. Environmental changes and lipid metabolism of higher plants. Physiol. Plant. 64:118-122.

Lyons, J.M. 1973. Chilling injury in plants. Annu. Rev. Plant Physiol. 24:445-466.

Mazliak, P. 1973. Lipid metabolism in plants. Annu. Rev. Plant Physiol. 24:287-310.

Quinn, P.J. 1981. The fluidity of cell membranes and its regulation. Progress Biophys. Molec. Biol. 38:1-104.

Radunz, A. 1986. On the function of methyl-branched chain fatty acids in phospholipids of cell membranes of higher plants, p. 197-200. In: P.K. Stumpf, J.B. Mudd, and W.D. Nes (eds.). The metabolism, structure, and function of plant lipids. Proc. 7th Intl. Symp. of Plant Lipids. Davis, Calif.

Raison, J.K. and E.A. Chapman. 1976. Membrane phase changes in chilling sensitive Vigna radiata and their significance to growth. Austral. J. Plant Physiol. 3:291-299.

Sandermann, H., Jr. 1978. Regulation of membrane enzymes by lipids. Biochim. Biophys. Acts 515:209-237.

Sellden, G. and E. Selstam. 1976. Changes in chloroplast lipids during the development of photosynthetic activity in barely etio-chloroplasts. Physiol. Plant. 37:35-41.

Wang, C.Y. 1982. Physiological and biochemical responses of plants to chilling stress. HortScience 17:173-186.

Wang, S.Y. and M. Faust. 1988. Changes of fatty acids and sterols in apple buds during bud break induced by a plant bioregulator, thidiazuron. Physiol. Plant. 72:115-120.

Wang, S.Y. and M. Faust. 1989a. Nitroguanidines induce bud break and change sterol content in apple. J. Plant Growth Regulat. 8:143151.

Wang, S.Y. and M. Faust. 1989b. Changes of membrane polar lipids associated. with bud break in apple induced by nitroguanidines. J. Plant Growth Regulat. 8: 153-161.

Wang, S. Y., T. Sun, B.D. Whitaker, and M. Faust. 1988. Effect of paclobutrazol on membrane lipids in apple seedlings. Physiol. Plant. 73:560-564.

Williams, J. P., M.U. Khan, and K. Mitchell. 1983. Galactolipid biosynthesis in leaves of 16:3- and 18:3-plants, p. 28-39. In: W.W. Thomson, J.B. Mudd, and M. Gibbs (eds. ). Biosynthesis and function of plant lipids. Proc. 6th Annu. Symp. in Bet. Riverside, Calif. 